\title{
Low Prevalence of Campylobacteriosis in the Northern Region of India
}

\author{
Chetana Vaishnavi1 ${ }^{*}$, Meenakshi Singh1, Jarnail Singh Thakur², Babu Ram Thapa1 \\ ${ }^{1}$ Department of Gastroenterology, Postgraduate Institute of Medical Education and Research, Chandigarh, \\ India \\ ${ }^{2}$ Department of Community Medicine (School of Public Health), Postgraduate Institute of Medical Education \\ and Research, Chandigarh, India \\ Email: ${ }^{*}$ cvaishnavi@rediffmail.com, c.vaishnavi@rediffmail.com
}

Received 5 February 2015; accepted 25 February 2015; published 3 March 2015

Copyright (C) 2015 by authors and Scientific Research Publishing Inc.

This work is licensed under the Creative Commons Attribution International License (CC BY).

http://creativecommons.org/licenses/by/4.0/

(c) (i) Open Access

\begin{abstract}
Campylobacter is one of the most common bacterial enteropathogens of food borne origin in industrialized countries with $C$. jejuni being the most common species followed by $C$. coli. The prevalence of Campylobacters in and around Chandigarh, India was studied by phenotypic and genotypic methods. Fecal samples from 1145 diarrheal patients and 102 healthy subjects from hospital and community were cultured on Campylobacter media and identified by Gram stain, biochemical investigations and serotyping. Molecular identification of Campylobacter isolates was done using specific primers to unique regions of 165 rRNA, Campylobacter jejuni (hip0), Campylobacter coli $(\operatorname{aspK)}$, Campylobacter lari (glyA) and Campylobacter upsaliensis (IpxA) genes. Identification of specific genes to look for resistance to nalidixic acid, ciprofloxacin, tetracyclin and streptomycin was also done. Campylobacters were isolated from $2.6 \%$ of patients with diarrhea. Campylobacteriosis was more prevalent in children $\leq 5$ years old and during summer season. The most frequent serotypes were $\mathrm{S}: 27, \mathrm{~B}: 2, \mathrm{Z}_{5}: 52$ and $\mathrm{V}: 32$. All the Campylobacters isolated by culture were confirmed genotypically by identification of 16S rRNA, hipO and aspK genes. Of the 30 isolates, 27 were $C$. jejuni and 3 were $C$. coli. No $C$. lari or $C$. upsaliensis were detected. Antibiotic resistance was $\mathbf{4 0} \%$ for nalidixic acid, $23.3 \%$ for ciprofloxacin, $50 \%$ for tetracyclin and $20 \%$ for streptomycin. Campylobacter prevalence is low in the region with $C$. jejuni being the most common species. A high degree of resistance was found for nalidixic acid and tetracyclin but moderate for ciprofloxacin and streptomycin.
\end{abstract}

\section{Keywords}

Antibiotic Resistance, Campylobacter Diarrheas, Molecular Investigation, Phenotypes, Serotyping

\footnotetext{
"Corresponding author.
} 


\section{Introduction}

Campylobacter is an important cause of acute bacterial diarrhea worldwide. The most common species causing campylobacteriosis in human beings is C. jejuni (95\%) followed by C. coli (5\%). Other species like C. fetus, C. upsaliensis and $C$. lari rarely cause human infections. Most of the isolates causing human gastroenteritis are thermo-tolerant variety and can grow even at incubation temperatures of $42^{\circ} \mathrm{C}$. Campylobacter is generally transmitted to humans by domestic animals, unpasteurized milk and contaminated water. The infectious dose is very small and as little as 500 cells are sufficient to produce gastroenteritis. Campylobacteriosis is characterized by fever, bloody diarrhea, headache and severe abdominal pain. When diarrhea is minimal but abdominal pain is present, it can lead to a mistaken diagnosis of acute abdomen and subsequently unnecessary laparotomy. Fever persisting up to 1 week is reported by $>90 \%$ of the patients. However, $50 \%$ of persons infected with Campylobacters are asymptomatic [1].

Most Campylobacter infections are sporadic in nature but some outbreaks have also been reported at times [2]. There is a seasonal peak for Campylobacter infections. In temperate climates, the incidence of infection is roughly twice as high in summer as in winter, and the incidence is high in rural than in urban communities [2]. Campylobacter is isolated from infants and young adults more frequently than from persons in other age groups and more frequently from males than from females [3]. Most people who develop campylobacteriosis recover completely within 2 - 5 days, but in some cases recovery can take up to 10 days [4]. Common long term complications of campylobacteriosis are Guillain Barré syndrome, rheumatoid arthritis, bacteremia and inflammatory bowel disease. Local complications of Campylobacter infections occur as a result of direct spread from gastrointestinal tract and can include cholecystitis, pancreatitis, peritonitis [4], massive gastrointestinal hemorrhage [5], thyroiditis [6] and prosthetic joint infection [7]. Timely treatment can reduce the duration and severity of the infection and reduce the chances of serious complications.

Campylobacteriosis is estimated to affect over 2.4 million persons annually, or $0.8 \%$ of the population [3] making it a highly important organism from a socio-economic perspective but many of the cases go undiagnosed. It is envisaged that campylobacteriosis has been in existence for many years but has only recently been recognized as a common infection due to improved laboratory methods. As Campylobacter is a major health burden for both developed and developing countries, Campylobacter infections remain a high research priority in order to improve the strategies for prevention and management of the disease. The present work was carried out to study the prevalence of campylobacteriosis in and around Chandigarh, which is in the northern region of India.

\section{Methods}

The study was approved by the Institute Ethical Committee and was conducted between May 2009 and January 2013.

\subsection{Study Population}

Both adult and paediatric patients with complaints of diarrhea were investigated. They included outpatients and inpatients from wards of hospitals in Chandigarh and those situated in slum areas of various colonies. Patients from community areas like Mauli Jagran and Indira Colony in the region around Chandigarh, India were also investigated by door-to-door visits. A total of 1145 patients were enrolled and informed, consent was taken from all the patients or their wards involved in the study. Detailed enquiry regarding their clinical symptoms was recorded in a pre-printed proforma. Healthy subjects $(n=102)$ from among the attendants of patients, who had no diarrhea of any kind, were also included in the study.

\subsection{Collection and Transportation of the Fecal Samples to the Laboratory}

Sterile stool containers (Stericol, Himedia, India) with $3 \mathrm{ml}$ of Campy-thioglycollate media were given to patients and healthy subjects. They were instructed to collect approximately 4 - 5 grams of the stool sample especially from mucoid or bloody area and transport the same immediately to the Microbiology Division of the department for processing. Fecal samples from community were collected by door-to-door visit by the research staff and transported in an ice-bucket to the laboratory. The samples were collected from the community either on the same day of visit or the next day depending on the frequency of diarrhea in the cases. 


\subsection{Culture for Campylobacters}

The stool samples were inoculated directly either on selective Campylobacter agar base media containing vancomycin, polymyxin B, trimethoprim and 5\% - 7\% defibrinated sheep blood or charcoal cefoperazone deoxycholate agar by quadrant streaking. For culture using filtration technique, stool samples were homogenized in physiological saline as 1:1 ratio and the homogenate was passed through membrane filters $(0.45 \mu \mathrm{m}$ and 0.65 $\mu \mathrm{m})$ placed on media plates. The filters were removed after an hour and discarded. The plates were incubated under microaerophilic conditions at $37^{\circ} \mathrm{C}$ and $42^{\circ} \mathrm{C}$ for $72 \mathrm{~h}$.

\subsection{Phenotypic Identification of Campylobacters}

Predominant or pure growth of grey to ceramic colonies on culture media were picked up and identified for Campylobacters as oxidase positive, catalase producing gram negative spiral rods. Campylobacter diarrhea was suspected when the isolate gave positive reaction with oxidase and/or hippurate in addition to any other positive identification tests. Serotyping of Campylobacter isolates was done based on the Penner scheme by the hemagglutination technique for heat stable antigens of $C$. jejuni using 25 C. jejuni specific antisera (Denka-Seiken, Japan) against the following heat-stable serotypes: $(1,44), 2,3,(4,13,16,43,50), 5,(6,7), 8,10,11,12,15,18$, $19,21,(23,36,53), 27,31,32,37,38,41,45,52,55$, and 57 with grouped antisera given in parentheses.

\subsection{Molecular Identification of Campylobacter Isolates and Antibiotic Resistance}

Molecular identification of Campylobacter isolates was done using specific primers to the unique regions of Campylobacter genus and different species as well as detection of resistance to antibiotics after isolation of DNA as follows.

\subsubsection{DNA Isolation}

For DNA isolation, a heavy growth of Campylobacter was suspended in Tris-EDTA buffer and boiled at $100^{\circ} \mathrm{C}$ for 10 mins. The boiled suspension was immediately transferred to an ice bath and incubated for $1 \mathrm{~h}$. Next, the suspension was centrifuged at 10,000 rpm for 2 mins and the supernatant was separated and checked for DNA by $0.8 \%$ agarose gel electrophoresis. DNA was also extracted from whole stool samples by QIAmp DNA mini kit (Qiagen, Netherlands) to tap any Campylobacter that could have been inadvertently lost.

\subsubsection{Polymerase Chain Reaction for Campylobacter Identification}

Polymerase chain reaction (PCR) assay was done using specific primers to the unique regions of Campylobacter genus and to the unique regions of different Campylobacter species. For genus identification of Campylobacter, 16S rRNA was amplified using C412F and C1228R primers. Species-specific identification of C. jejuni, C. coli, $C$. lari and $C$. upsaliensis was done by amplifying the hippuricase $(h i p O)$ gene, the aspartokinase $(a s p \mathrm{~K})$ gene, the serine hydroxymethyltransferase (glyA) gene and lipopolysaccharide (lpxA) gene respectively (Table 1).

\subsubsection{Polymerase Chain Reaction for Detection of Antibiotic Resistance}

Nalidixic acid resistance was identified by amplifying specific gyrA gene by PCR using primers gyrA F and gyrA R. Ciprofloxacin (gyrA) resistance in Campylobacter isolates was investigated by Mismatch Amplification Mutation Assay (MAMA) using a conserved forward primer, Campy MAMA gyrA1 and a mutation detection reverse primer, Campy MAMA gyrA5. An annealing temperature of $50^{\circ} \mathrm{C}$ was used to give 265 bp products which indicated the presence of the Thr-6 to Ile (ACA $\rightarrow$ ATA) mutation in the Campylobacter gyrA gene. Tetracyclin resistance was detected by using specific primers to amplify tetO gene. Streptomycin resistance was detected by amplifying the strA gene. All the PCR products were analyzed by electrophoresis on $1.8 \%$ agarose gel containing $0.1 \mu \mathrm{g} / \mathrm{ml}$ ethidium bromide.

\subsection{Statistical Analysis}

Data was analyzed by SPSS version 16.0. 
Table 1. Primers used for molecular identification of Campylobacter and antibiotic resistance genes.

\begin{tabular}{|c|c|c|}
\hline S. No. & Target Gene & Primer Sequence \\
\hline 1 & 16S rRNA & $\begin{array}{l}\text { F- 5' AATCTAATGGCTTAACCATTA 3', } \\
\text { R- 5' GTAACTAGTTTAGTATTCCGC 3' }\end{array}$ \\
\hline 2 & Hippuricase (hipO) (C. jejuni) & $\begin{array}{l}\text { F- 5'-GGAGAGGGTTTGGGTGGT-3' } \\
\text { R- 5'-AGCTAGCCTCGCATAATAACTTG-3' }\end{array}$ \\
\hline 3 & Aspartokinase (aspK) (C. coli) & $\begin{array}{l}\text { F- 5' GGTATGATTTCTACAAAGCGAG-3' } \\
\text { R- 5' ATAAAAGACTATCGTCGCGTG-3 }\end{array}$ \\
\hline 4 & glyA (C.lari) & $\begin{array}{l}\text { F- 5' TAGAGAGATAGCAAAAGAGA 3' } \\
\text { R- 5' TACACATAATAATCCCACCC 3' }\end{array}$ \\
\hline 5 & IpxA (C. upsaliensis) & $\begin{array}{l}\text { F- 5' CGATGATGTCAAATTGAAGC 3' } \\
\text { R- 5' TTCTAGCCCCTTGCTTGATG 3' }\end{array}$ \\
\hline 6 & Tetracycline (tetO) & $\begin{array}{l}\text { F- 5' AACTTAGGCATTCTGGCTCAC 3' } \\
\text { R-5' TCCCACTGTTCCATATCGTCA 3' }\end{array}$ \\
\hline 7 & Nalidixic acid (gyrA) & $\begin{array}{l}\text { F- 5' GCT CTT GTT TTA GCT TGATGCA 3' } \\
\text { R- 5' TTG TCG CCA TC CTA CAGCTA 3' }\end{array}$ \\
\hline 8 & Ciprofloxacin (MAMA gyrA) & $\begin{array}{l}\text { F- 5’ TTT TTA GCA AAG ATT CTG AT 3', } \\
\text { R- 5’CAA AGC ATC ATA AAC TGC AA 3', }\end{array}$ \\
\hline 9 & Streptomycin (strA) & $\begin{array}{l}\text { F- 5' CCAATCGCAGATAGAAGGCAAG 3', } \\
\text { R- 5' ATCAACTGGCAGGAGGAACAGG 3' }\end{array}$ \\
\hline
\end{tabular}

\section{Results}

There were 734 males and 411 females with age range of 17 days old to 87 years old in the diarrheal patient group. The clinical symptoms present in them at the time of examination were diarrhea in 959 (83.75\%), fever in 469 (40.96\%) and abdominal pain in 406 (35.45\%). Of the 102 age-matched healthy control subjects, there were 69 males and 33 females with age range of 2 months old to 77 years old.

\subsection{Phenotypic Methods}

Of the 1145 stool samples from diarrheic cases, Campylobacter was isolated from 30 (2.6\%) of them, but none from the control samples. Campylobacter positivity was found in 19 male patients and 11 female patients. The preponderance of infection was more in summer season (76.7\%) than in winter season (23.3\%). Fifty percent prevalence was seen in children $\leq 5$ years old and the remaining 50\% were scattered in the age group 10 - 66 years old with predominant positivity between 29 - 40 years old. The clinical symptoms fever and abdominal pain were found to be statistically significant $(\mathrm{p}=0.000)$ in the Campylobacter positive patients compared to the negative ones. With the serotyping of Campylobacter isolates, 93.3\% reacted to multiple antisera and thus belonged to multiple serotypes (Table 2). Two of the Campylobacter isolates were non-typeable. The most frequent serotypes in descending order were Groups $\mathrm{S}: 27, \mathrm{~B}: 2, \mathrm{Z}_{5}: 52$ and V:32.

\subsection{Molecular Methods}

All the Campylobacters isolated by culture (100\%) were also found to be positive for genus Campylobacter by PCR based on detection of species-specific 16S rRNA (Figure 1). Of the 30 Campylobacter isolates, 27 were $C$. jejuni and 3 were $C$. coli based on detection of hipO gene (Figure 2) and aspK gene (Figure 3) respectively. No co-infection of $C$. jejuni and $C$. coli were seen. However, molecular investigation of DNA from isolates and from whole fecal samples were found to be negative for glyA gene (C. lari) and lpxA gene (C. upsaliensis) depicting the absence of these two species. Antibiogram resistance study showed $40 \%(12 / 30)$ of the isolates resistant to nalidixic acid (Figure 4(a)), 23.3\% (7/30) to ciprofloxacin (Figure 4(b)), 50\% (15/30) to tetracyclin (Figure 5(a)) and 20\% (6/30) to streptomycin (Figure 5(b)). 
Table 2. Number of $C$. jejuni isolated showing multiple serotypes in alphabetical order.

\begin{tabular}{|c|c|}
\hline Serotypes & $\begin{array}{c}\text { No. of } C \text {. jejuni isolates showing } \\
\text { multiple serotypes }\end{array}$ \\
\hline A: 1,44 & 4 \\
\hline $\mathrm{B}: 2$ & 14 \\
\hline C:3 & 3 \\
\hline $\mathrm{D}: 4,13,16,43,50$ & 3 \\
\hline E:5 & 9 \\
\hline $\mathrm{F}: 6,7$ & 7 \\
\hline G:8 & 3 \\
\hline $\mathrm{I}: 10$ & 4 \\
\hline $\mathrm{J}: 11$ & 4 \\
\hline $\mathrm{K}: 12$ & 4 \\
\hline L:15 & 6 \\
\hline $\mathrm{N}: 18$ & 7 \\
\hline O:19 & 6 \\
\hline $\mathrm{P}: 21$ & 1 \\
\hline $\mathrm{R}: 23,36,53$ & 7 \\
\hline S:27 & 13 \\
\hline U:31 & 3 \\
\hline V:32 & 10 \\
\hline Y:37 & 3 \\
\hline Z:38 & 7 \\
\hline $\mathrm{Z}_{2}: 41$ & 5 \\
\hline $\mathrm{Z}_{4}: 45$ & 1 \\
\hline$Z_{5}: 52$ & 11 \\
\hline $\mathrm{Z}_{6}: 55$ & 2 \\
\hline $\mathrm{Z}_{7}: 57$ & 8 \\
\hline
\end{tabular}

Footnote: The most frequent serotypes are marked in bold.

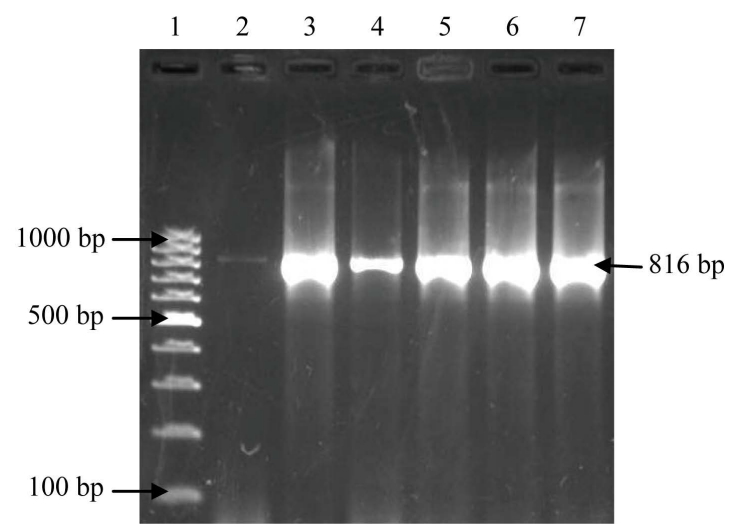

Figure 1. PCR detection of 16S rRNA gene (816 bp) of $C$. jejuni. Lane 1-100 bp Marker; Lane 2-Positive control; Lane 3 to 12Campylobacter isolates. 


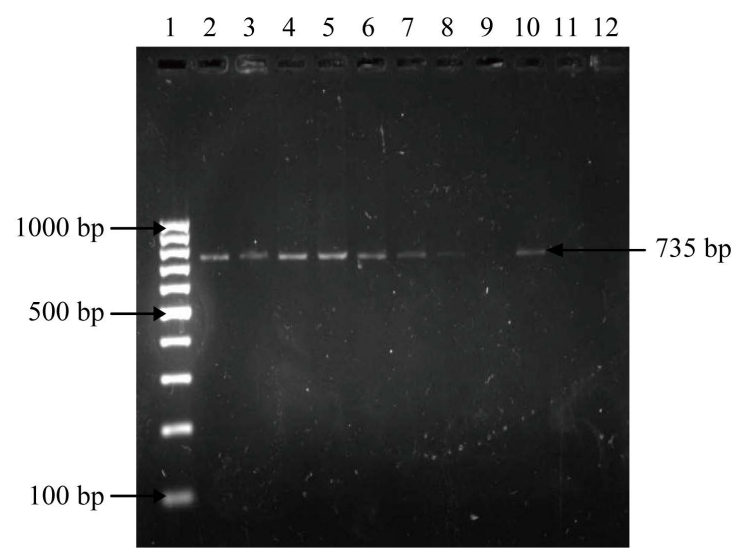

Figure 2. PCR detection of hipO gene (735 bp) of $C$. jejuni. Lane 1-100 bp Marker; Lane 2-Positive control; Lanes 3 to 5-Campylobacter isolates; Lane 12-Negative control.

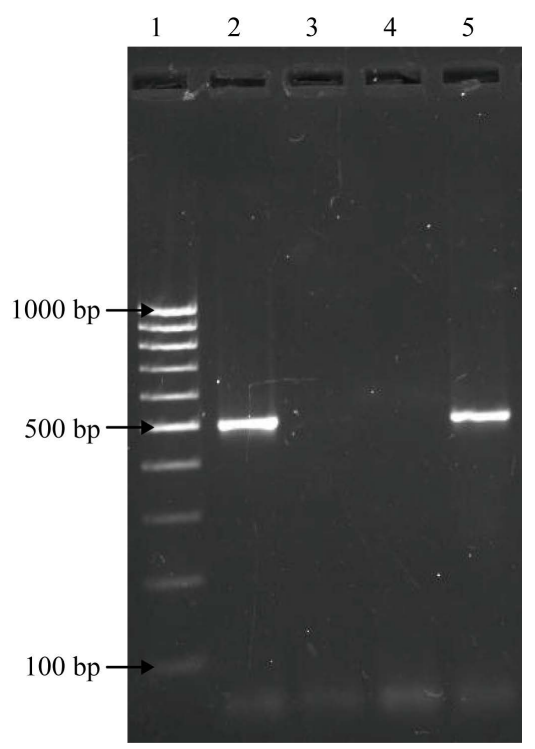

Figure 3. aspK gene of C. coli (500 bp). Lane 1-100 bp Marker; Lane 2-Positive control; Lanes 3 to 5Campylobacter isolates.

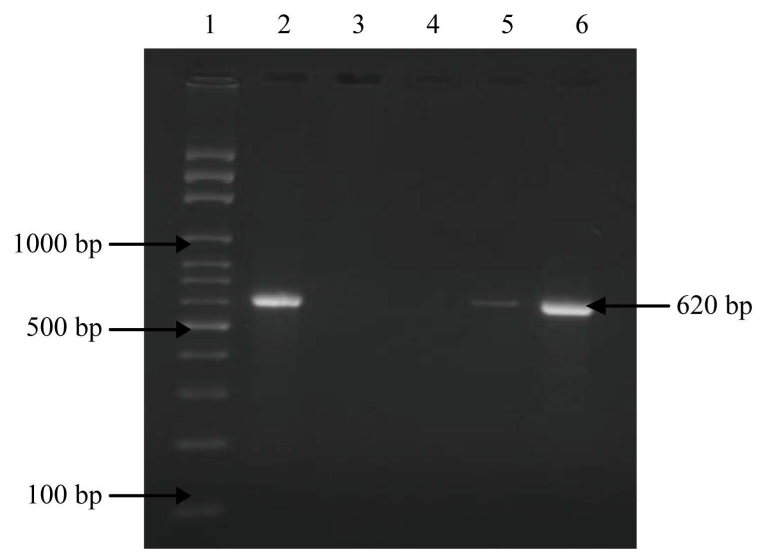

(a)

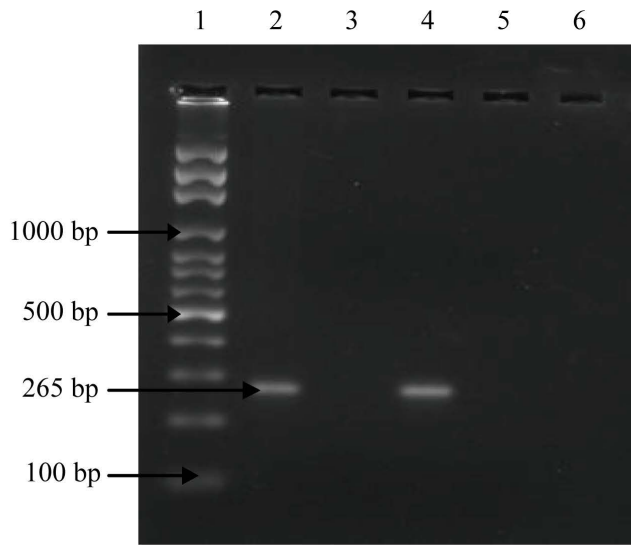

(b)

Figure 4. PCR detection of gyrA genes for resistance of nalidixic acid (620 bp) and ciprofloxacin (265 bp) respectively. Lane 1-Marker; Lane 2-Positive control; Lanes 3 to 6-Campylobacter isolates. 


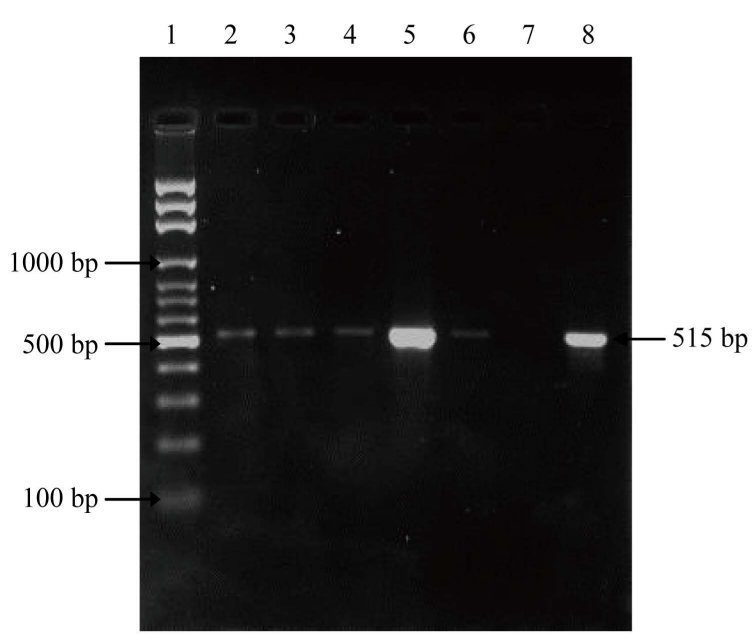

(a)

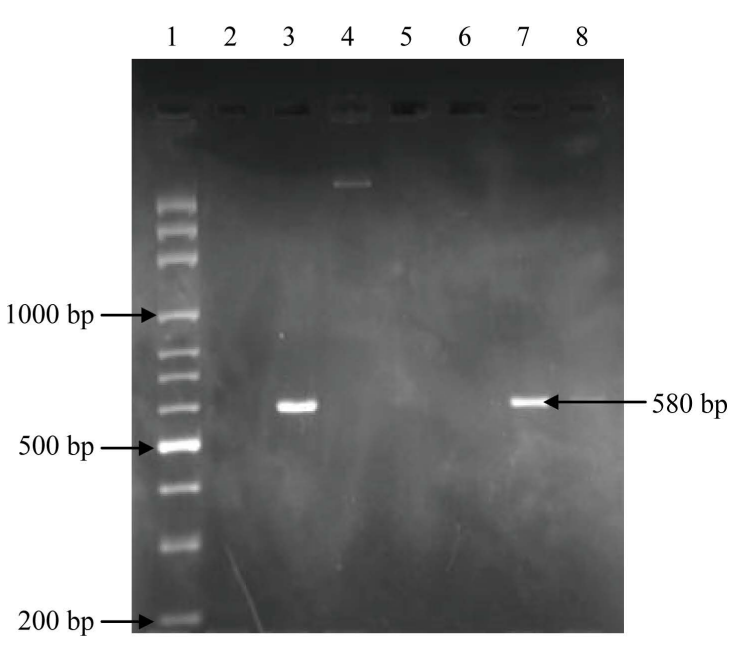

(b)

Figure 5. PCR detection of tetO and strA genes for resistance of tetracycline (515 bp) and streptomycin (580 bp) respectively. (a) Lane 1-100 bp Marker; Lane 2-Positive control; Lanes 3 to 8-Campylobacter isolates; (b) Lane 1-Positive control; Lane 2 Negative control; Lane 3 Positive control; Lanes 4 to 8-Campylobacter isolates.

\section{Discussion}

Although 14 Campylobacter species have been identified, in the United States $>99 \%$ of reported infections with Campylobacter are with C. jejuni [8]. The frequency of isolation of $C$. jejuni in various parts of the world varies due to the varying standards of living conditions, water supply and feeding habits. The isolation rate of Campylobacter reported from different regions of the world are 9.5\% (France) [9], 6.7\% (United Kingdom) [10], 9\% (Central Africa) [11], 44\% (South Africa) [12], 17.7\% Bangladesh [13], 8\% (Tehran) [14], 12\% (Lahore) [15], and 18\% (Rawalpindi) [16]. Reports from India include 16\% from rural population in Mumbai, $8.6 \%$ from Ranchi [17] and 14.8\% from Vellore [18]. Salim et al. [3] isolated Campylobacter in 10\% of the 50 fecal samples investigated from children suffering from diarrhea, dysentery or acute gastroenteritis. In the present study, Campylobacters were isolated in only 2.6\% samples from hospital and community and none from 102 healthy subjects from the same community. This shows a very low level of prevalence of Campylobacter in the region and can be attributed to availability of good drinking water sources and better hygiene practices. However, interestingly, the patients with Campylobacter positivity also had significant clinical symptoms of fever and abdominal pain compared to those who were Campylobacter negative.

Campylobacter more frequently affects children. In tropical developing countries, Campylobacter infections are hyperendemic among young children especially those aged $>2$ years old. Salim et al. [3] reported the minimum age for Campylobacter isolation is 2 days old and the maximum is 4 years old respectively. The minimum and maximum age of isolation reported from Rawalpindi was 3 months old and 48 months old. Studies from Ranchi [17] and Vellore [18] showed maximum isolation from $>6$ years old and from pre-school children respectively. In the present study, the minimum age of isolation of Campylobacters was 5 months old and the maximum was 66 years old with $50 \%$ prevalence in children $\leq 5$ years old, especially those living in rural regions of Chandigarh. In the remaining 50\% predominant positivity was seen in patients aged 29 - 40 years old.

In industrialized nations, there is a preponderance of males among Campylobacter infected persons [19], the reasons for which are unknown. In the present study also, Campylobacter infections were more common in males than in females. Campylobacteriosis occurs much more frequently in summer than in winter [20]. In the present study also a 3-fold higher frequency of Campylobacter diarrhea was seen during the summer seasons.

Outbreaks of Campylobacters have been reported from several countries. Yu et al. [21] reported the first recognized major $C$. jejuni outbreak was associated with contaminated chicken among middle school students in Korea where an attack rate of $11.6 \%$ occurred with $40.3 \%$ stool samples positives for $C$. jejuni. Karagiannis et al. [5] reported a C. jejuni outbreak in Crete in which most cases originated from rural areas and had a strong epidemiological evidence that tap water was the vehicle of the outbreak. Longenberger et al. [22] reported a multistate outbreak of Campylobacter infections was associated with unpasteurized milk in US which resulted in 
148 illnesses. Taylor et al. [23] reviewed reports of campylobacteriosis in US from 1997 to 2008 where 262 outbreaks with 9135 illnesses, 159 hospitalizations and 3 deaths were recorded. Though sporadic infection of Campylobacter has been observed from time to time in India, no outbreak as such has been reported.

In our study, C. jejuni was present in $90 \%$ and C. coli in $10 \%$ of the patient samples with no co-infection seen amongst them. Both C. lari and C. upsaliensis were not found among the isolates. The prevalence of a particular serotype differs across countries based on their geographic locations. In Japan, the most predominant serotypes are B, D, and L, while those in Denmark are serotypes B, A, and D [24] [25]. Ishihara et al. [26] identified 18 serotypes with major serotypes being $\mathrm{B}, \mathrm{D}$ and $\mathrm{R}$ among the $C$. jejuni isolates from humans. In another study from Thailand [27], C. jejuni isolates from humans were classified into 10 Penner serotypes viz. B, C, R, E, G, A, K, D, I and L. The most predominant serotype was B (9 strains), followed by serotype C (8 strains), R (5 strains), E (4 strains), G (3 strains), A (2 strains), K (2 strains), D (1 strain), I (1 strain), and L (1 strain). In the present study, the most frequent serotypes were S:27, B:2, Z $5: 52$ and V:32 in descending order. Serotype B appears to be present everywhere as observed from reported studies.

Most Campylobacter diarrhea is generally self-limiting, but antimicrobial treatment may be required for patients with severe, prolonged or systemic infections or to control infection in high-risk groups. The antimicrobial agents of choice are macrolides and fluoroquinolones. However, antibiotic management of Campylobacter has become more complex as $C$. jejuni has undergone a rapid increased resistance to fluoroquinolone [28] due to their use in food animals [4]. The clinical impact of antibiotic resistance in Campylobacter infections are reported in epidemiological studies. Patients with quinolone-resistant $C$. jejuni, treated with fluoroquinolones in Minnesota in 1997 had a median duration of diarrhea of 10 days compared to 7 days in those with sensitive strains [29]. Gupta et al. [30] reported 31\% patients with ciprofloxacin-resistant Campylobacter were hospitalized for gastroenteritis compared to 3\% patients with sensitive strains. In a case control study carried out in 1998-1999 in 290 patients who did not take anti-diarrheal medication for campylobacteriosis, those with ciprofloxacin-resistant strains had a mean duration of diarrhea of 9 days, compared to 7 days in those with sensitive isolates [31]. Of 85 persons who took fluoroquinolone antimicrobials only, diarrhea lasted a mean of 8 days in those with resistant strains but 6 days in those with sensitive isolates. In 63 patients who took no antimicrobials, those with ciprofloxacin-resistant isolates had diarrhea for a mean of 12 days versus 6 days for sensitive strains. This suggests that those infected with resistant Campylobacters have a prolonged and more severe infection than those with sensitive strains.

Goodchild et al. [32] in a study of 200 Campylobacters isolates from New Zealand, reported resistance to ciprofloxacin in $4 \%$ of the $C$. jejuni isolates against $20 \%$ resistance in "non-jejuni" isolates, with only one of the isolates being resistant to tetracyclin. Korolik et al. [33] studied antibiotic profile on 81 strains of $C$. jejuni and 8 of C. coli collected from 1989-1990 and 79 of C. jejuni and 6 of C. coli collected from 1994-1995. During the second period, doxycycline resistance fell from $10 \%$ to $2.5 \%$ in C. jejuni and from $25 \%$ to $16 \%$ in C. coli. This could be because though tetracyclins help in the treatment of clinical campylobacteriosis, in practice they are rarely used as the tetO gene has shown to confer extremely high-levels of tetracyclin resistance [34]. Huysman and Turnidge [35] in a study of 100 strains (79 C. jejuni, 19 C. coli and 2 C. lari) found all strains were sensitive to erythromycin, gentamicin, nalidixic acid and ciprofloxacin and 9 strains resistant to tetracyclin. A study in the Hunter region of New South Wales of Australia investigating resistance of 180 isolates of C. jejuni found 11\% of isolates resistant to tetracyclin, $3.4 \%$ to nalidixic acid, $2.9 \%$ to ciprofloxacin, $64 \%$ to ampicillin, $3.4 \%$ to erythromycin and $48 \%$ to roxithromycin with none resistant to gentamicin [36].

In the present study, antibiotic resistance was $40.0 \%$ for nalidixic acid, $23.3 \%$ for ciprofloxacin, $50 \%$ for tetracyclin and $20 \%$ for streptomycin, indicating the development of multiple resistances. Molecular techniques such as MAMA-PCR [37] successfully detect ciprofloxacin-resistant $C$. jejuni and $C$. coli isolates by identifying a mutation Thr-86-Ile in the gyrA gene, commonly associated with ciprofloxacin resistance in Campylobacters. However, the major disadvantage of this technique is that it may not be able to detect resistance if a new, unexpected resistance mechanism occurs [38].

\section{Conclusion}

Antibiotic resistance rates are rising worldwide with the development of multiple resistances to several classes of antibiotics. Therefore, the decision to use antibiotics should be made judiciously. Routine use of antibiotic pro- phylaxis to prevent Campylobacter infections is not recommended. From the study, it is concluded that $C$. 
jejuni is most commonly isolated among the Campylobacter diarrheas with high degree of resistance for nalidixic acid and tetracyclin and moderate degree of resistance for ciprofloxacin and streptomycin.

\section{Acknowledgements}

Mr. Prashant Kapoor is acknowledged for his technical support. We are grateful to Dr. Ajay Prakash Patel for statistical analysis.

\section{Funding}

This work was supported by the Indian Council of Medical Research, New Delhi.

\section{Transparency Declaration}

There are no conflicts of interest to report.

\section{References}

[1] Calva, J.J., Ruiz-Palacioz, G.M., Lopez-Vidal, A.B., Ramos, A. and Bojalil, R. (1988) Cohort Study of Intestinal Infection with Campylobacter in Mexican Children. The Lancet, 1, 503-506. http://dx.doi.org/10.1016/S0140-6736(88)91297-4

[2] Vaishnavi, C. (2013) Clinical Manifestations of Campylobacter Infections. In: Vaishnavi, C., Ed., Infections of the Gastrointestinal System, Jaypee Brothers, New Delhi, 263-283. http://dx.doi.org/10.5005/jp/books/11918

[3] Salim, S.M., Mandal, J. and Parija, S.C. (2014) Isolation of Campylobacter from Human Stool Samples. Indian Journal of Medical Microbiology, 32, 35-38. http://dx.doi.org/10.4103/0255-0857.124294

[4] Allos, B.M. (2001) Campylobacter jejuni Infections: Update on Emerging Issues and Trends. Clinical Infectious Diseases, 32, 1201-1206. http://dx.doi.org/10.1086/319760

[5] Karagiannis, I., Sideroglou, T., Gkolfinopoulou, K., Tsouri, A., Lampousaki, D., Velonakis, E.N., Scoulica, E.V., Mellou, K., Panagiotopoulos, T. and Bonovas, S. (2010) A Waterborne Campylobacter jejuni Outbreak on a Greek Island. Epidemiology and Infection, 138, 1726-1734. http://dx.doi.org/10.1017/S0950268810002116

[6] Diggins, B., Diaz-Cano, S.J. and Schulte, K.M. (2014) Campylobacter jejuni as a Cause of Acute Infectious Thyroiditis, on a Background of SLE-Related End Stage Renal Failure and CMV Viraemia: A Case Report and Review of the Literature. Journal of Vaccines \& Vaccination, 5, 229.

[7] Vasoo, S., Schwab, J.J., Cunningham, S.A., Robinson, T.J., Cass, J.R., Berbari, E.F., Walker, R.C., Osmon, D.R. and Patel, R. (2014) Campylobacter Prosthetic Joint Infection. Journal of Clinical Microbiology, 52, 1771-1774. http://dx.doi.org/10.1128/JCM.03572-13

[8] Ramirez-Hernandes, A. (2014) Adherence Inhibition of Human Pathogen Campylobacter jejuni and Campylobacter coli by Non-Digestible Oligo sachharides. Dissertations \& Theses in Food Science and Technology, University of Nebraska-Lincoln, Lincoln. http://digitalcommons.unl.edu/cgi/viewcontent.cgi?article=1048\&context=foodscidiss

[9] Bessède, E., Delcamp, A., Sifré, E., Buissonnière, A. and Mégraud F. (2011) New Methods for Detection of Campylobacters in Stool Samples in Comparison to Culture. Journal of Clinical Microbiology, 49, 941-944. http://dx.doi.org/10.1128/JCM.01489-10

[10] Linton, D., Lawson, A.J., Owen, R.J. and Stanley, J. (1997) PCR Detection, Identification to Species Level, and Fingerprinting of Campylobacter jejuni and Campylobacter coli Direct from Diarrheic Samples. Journal of Clinical Microbiology, 35, 2568-2572.

[11] De Mol, P. and Bosmans, E. (1978) Campylobacter Enteritis in Central Africa. The Lancet, 311, 604. http://dx.doi.org/10.1016/S0140-6736(78)91044-9

[12] Bokkenheuser, V.D., Richardson, N.J., Bryner, J.H., Roux, D.J., Schutte, A.B., Koornhof, H.J., Freiman, I. and Hartman, E. (1979) Detection of Enteric Campylobacteriosis in Children. Journal of Clinical Microbiology, 9, $227-232$.

[13] Blaser, M.J., Glass, R.I., Huq, M.I., Stoll, B., Kibriya, G.M. and Alim, A.R. (1980) Isolation of Campylobacter Fetus subsp. jejuni from Bangladeshi Children. Journal of Clinical Microbiology, 12, 744-747.

[14] Feizabadi, M.M., Dolatabadi, S. and Zali, M.R. (2007) Isolation and Drug-Resistant Patterns of Campylobacter Strains Cultured from Diarrheic Children in Tehran. Japanese Journal of Infectious Diseases, 60, 217-219.

[15] Khalil, K., Lindblom, G.B., Mazhar, K., Khan, S.R. and Kajiser, B. (1993) Early Child Health in Lahore, Pakistan: VIII. Microbiology. Acta Paediatrica, 82, 87-94. http://dx.doi.org/10.1111/j.1651-2227.1993.tb12909.x

[16] Ali, A.M., Qureshi, A.H., Rafi, S., Roshan, E., Khan, I., Malik, A.M. and Shahid, S.A. (2003) Frequency of Campylo- 
bacter jejuni in Diarrhea/Dysentery in Children in Rawalpindi and Islamabad. Journal of the Pakistan Medical Association, 53, 517-520.

[17] Rizal, A., Kumar, A. and Vidhyarthi, A.S. (2010) Prevalence of Pathogenic Genes in Campylobacter jejuni Isolated from Poultry and Humans. Internet Journal of Food Safety, 12, 29-34.

[18] Rajan, D.P. and Mathan, V.I. (1982) Prevalence of Campylobacter Fetus subsp. jejuni in Healthy Populations in Southern India. Journal of Clinical Microbiology, 15, 749-751.

[19] Friedman, C.R., Neimann, J., Wegener, H.C. and Tauxe, R.V. (2000) Epidemiology of Campylobacter jejuni Infections in the United States and Other Industrialized Nations. Campylobacter, 2, 121-138.

[20] Schielke, A., Rosner, B.M. and Stark, K. (2014) Epidemiology of Campylobacteriosis in Germany-Insights from 10 Years of Surveillance. BMC Infectious Diseases, 14, 30. http://dx.doi.org/10.1186/1471-2334-14-30

[21] Yu, J.H., Kim, N.Y., Cho, N.G., Kim, J.H., Kang, Y.A. and Lee, H.G. (2010) Epidemiology of Campylobacter jejuni Outbreak in a Middle School in Incheon, Korea. Journal of Korean Medical Science, 25, 1595-1600. http://dx.doi.org/10.3346/jkms.2010.25.11.1595

[22] Longenberger, A.H., Palumbo, A.J., Chu, A.K., Moll, M.E., Weltman, A. and Ostroff, S.M. (2013) Campylobacter jejuni Infections Associated with Unpasteurized Milk-Multiple States, 2012. Clinical Infectious Diseases, 57, $263-266$. http://dx.doi.org/10.1093/cid/cit231

[23] Taylor, E.V., Herman, K.M., Ailes, E.C., Fitzgerald, C., Yoder, J.S., Mahon, B.E. and Tauxe, R.V. (2013) Common Source Outbreaks of Campylobacter Infection in the USA, 1997-2008. Epidemiology and Infection, 141, 987-996. http://dx.doi.org/10.1017/S0950268812001744

[24] Nielsen, E.M., Engberg, J., Fussing, V., Petersen, L., Brogren, C.H. and On, S.L. (2000) Evaluation of Phenotypic and Genotypic Methods for Subtyping of Campylobacter jejuni Isolates from Humans, Poultry, and Cattle. Journal of Clinical Microbiology, 38, 3800-3810.

[25] Saito, S., Yatsuyanagi, J., Harata, S., Ito, Y., Shinagawa, K., Suzuki, N., Amano, K. and Enomoto, K. (2005) Campylobacter jejuni Isolated from Retail Poultry Meat, Bovine Feces and Bile, and Human Diarrheal Samples in Japan: Comparison of Serotypes and Genotypes. FEMS Immunology \& Medical Microbiology, 45, 311-319. http://dx.doi.org/10.1016/j.femsim.2005.05.006

[26] Ishihara, K., Yamamoto, T., Satake, S., Takayama, S., Kubota, S., Negishi, H., Kojima, A., Asai, T., Sawada, T., Takahashi, T. and Tamura, Y. (2006) Comparison of Campylobacter Isolated from Humans and Food-Producing Animals in Japan. Journal of Applied Microbiology, 100, 153-160. http://dx.doi.org/10.1111/j.1365-2672.2005.02769.X

[27] Boonmar, S., Yingsakmongkon, S., Songserm, T., Hanhaboon, P., Passadurak, W., et al. (2007) Serotypes, Antimicrobial Susceptibility, and gyr A Gene Mutation of Campylobacter jejuni Isolates from Humans and Chickens in Thailand. Microbiology and Immunology, 51, 531-537. http://dx.doi.org/10.1111/j.1348-0421.2007.tb03941.x

[28] Kirkpatrick, B.D. and Tribble, D.R. (2011) Update on Human Campylobacter jejuni Infections. Current Opinion in Gastroenterology, 27, 1-7. http://dx.doi.org/10.1097/MOG.0b013e3283413763

[29] Smith, K.E., Besser, J.M., Hedberg, C.W., Leano, F.T., Bender, J.B., Wicklund, J.H., Johnson, B.P., Moore, K.A. and Osterholm, M.T. (1999) Quinolone-Resistant Campylobacter jejuni Infections in Minnesota, 1992-1998. New England Journal of Medicine, 340, 1525-1532. http://dx.doi.org/10.1056/NEJM199905203402001

[30] Gupta, A., Nelson, J.M., Barrett, T.J., Tauxe, R.V., Rossiter, S.P., Friedman, C.R., Joyce, K.W., Smith, K.E., Jones, T.F., Hawkins, M.A., Shiferaw, B., Beebe, J.L., Vugia, D.J., Rabatsky-Her, T., Benson, J.A., Root, T.P., Angulo, F.J. and NARMS Working Group (2004) Antimicrobial Resistance among Campylobacter Strains, United States, 1997-2001. Emerging Infectious Diseases, 10, 1102-1109. http://dx.doi.org/10.3201/eid1006.030635

[31] Nelson, J.M., Smith, K.E., Vugia, D.J., Rabatsky-Her, T., Segler, S.D., Kassenborg, H.D., Zansky, S.M., Joyce, K., Marano, N., Hoekstra, R.M. and Angulo, F.J. (2004) Prolonged Diarrhea Due to Ciprofloxacin-Resistant Campylobacter Infection. Journal of Infectious Diseases, 190, 1150-1157. http://dx.doi.org/10.1086/423282

[32] Goodchild, C., Dove, B., Riley, D. and Morris, A.J. (2001) Antimicrobial Susceptibility of Campylobacter Species. The New Zealand Medical Journal, 114, 560-561.

[33] Korolik, V., Chang, J. and Coloe, P.J. (1996) Variation in Antimicrobial Resistance in Campylobacter spp. Isolated in Australia from Humans and Animals in the Last Five Years. In: Newell, D.G., Ketley, J.M. and Feldman, R.A., Eds., Campylobacters, Helicobacters, and Related Organisms, Plenum Press, New York, 393-398.

[34] Gibreel, A., Tracz, D.M., Nonaka, L., Ngo, T.M., Connell, S.R. and Taylor, D.E. (2004) Incidence of Antibiotic Resistance in Campylobacter jejuni Isolated in Alberta, Canada, from 1999 to 2002, with Special Reference to tet(O)-Mediated Tetracycline Resistance. Antimicrobial Agents and Chemotherapy, 48, 3442-3450. http://dx.doi.org/10.1128/AAC.48.9.3442-3450.2004

[35] Huysman, M.B. and Turnidge, J.D. (1997) Disc Susceptibility Testing for Thermophilic Campylobacters. Pathology, 29, 209-216. http://dx.doi.org/10.1080/00313029700169884 
[36] Sharma, H., Unicombe, L., Forbes, W., Djordjevic, S., Valcanis, M., Dalton, C. and Ferguson, J. (2003) Antibiotic Resistance in Campylobacter jejuni Isolated from Humans in the Hunter Region, New South Wales. Communicable Diseases Intelligence Quarterly Report, 27, S80-S88.

[37] Zirnstein, G., Li, Y., Swaminathan, B. and Angulo, F. (1999) Ciprofloxacin Resistance in Campylobacter jejuni Isolates: Detection of gyrA Resistance Mutations by Mismatch Amplification Mutation Assay PCR and DNA Sequence Analysis. Journal of Clinical Microbiology, 37, 3276-3280.

[38] Fluit, A.C., Visser, M.R. and Schmitz, F.J. (2001) Molecular Detection of Antimicrobial Resistance. Clinical Microbiology Reviews, 14, 836-871. http://dx.doi.org/10.1128/CMR.14.4.836-871.2001

\section{List of Abbreviations}

1) aspK-aspartokinase

2) C. coli-Campylobacter coli

3) C. fetus-Campylobacter fetus

4) C. jejuni-Campylobacter jejuni

5) C. lari-Campylobacter lari

6) C. upsaliensis-Campylobacter upsaliensis

7) DNA-Deoxyribonucleic acid

8) EDTA-Ethylenediamine tetraacetic acid

9) gly A-Glycine

10) gyrA-Gyrase A

11) hipO-hippuricase

12) lpxA-lipopolysaccharide

13) MAMA-Mismatch Amplification Mutation Assay

14) PCR-Polymerase chain reaction

15) \%-Percentage

16) rRNA—Ribosomal ribonucleic acid 